\title{
Respiratory polymerase chain reaction - point of care test for patients with suspected influenza on the ambulatory care unit
}

\author{
Authors: Anuradha Jayachandran and Mahin Ahmad
}

\begin{abstract}
Aims
Availability of respiratory polymerase chain reaction $(P C R)$ result within 3 hours of the decision to do test and within 1 hour of sample collection to enable timely decision making for patients with suspected influenza.
\end{abstract}

\section{Background}

An audit from November 2016 to March 2017 demonstrated that respiratory PCR testing done via the lab takes on average 8 hours 44 minutes from the clinician's decision to do the test to actual sample collection. It also showed that on average it takes 3 hours and 18 minutes from sample collection to actual result availability.

This resulted in inappropriate isolation of patients, impaired patient flow coupled with poor staff and patient experience. There was also a delay in decision making as the clinician who decides the need for the test is often not the same clinician reviewing the result, due to several hours delay in processing the sample via the lab.

\section{Method}

Patients with suspected influenza were isolated as per local guidelines and nasal and throat swabs collected by health care assistants. A respiratory PCR test using a Cepheid (GeneXpert) machine was done after patient review by the acute medical unit consultants or advanced nurse practitioners.

\section{Results}

101 samples were collected from 20 November 2017 to 2 January 2018 from patients with suspected influenza. 38\% had positive PCR and $62 \%$ negative PCR. 14 patients had Flu A, 17 Flu B and seven respiratory syncytial virus.

$47 \%$ of the positive PCR patients were discharged, while the remaining patients were admitted for other medical reasons or modified national early warning scores $>$ or $=4$.

Authors: Acute medicine, Wirral University Teaching Hospital, Wirral, UK
The average time taken from the clinicians' decision to do the test to the availability of result for patients assessed on the ambulatory care unit was 2 hours 49 minutes. All patients had their PCR result within 1 hour of collection of sample due to near patient testing and avoidance of transportation delays to the lab.

\section{Conclusion}

All the aims of this project were achieved. During this trial, all patients with suspected influenza were isolated appropriately prior to the result availability compared with $47 \%$ in the previous year's audit. There was a greater than $75 \%$ reduction in time (compared with previous audit) from the clinicians' decision to do the test to the availability of result, leading to better flow of patients. The staff and patient experience improved due to earlier availability of results and subsequent prompt decision making. As a result of this project it has become clear that near patient testing for influenza is better than laboratory testing.

\section{Conflict of interest statement}

The authors listed hereby certify that they have no affiliations with or involvement in any organisation or entity with any financial interest (such as honoraria; educational grants; participation in speakers' bureaus; membership, employment, consultancies, stock ownership, or other equity interest; and expert testimony or patentlicensing arrangements), or non-financial interest (such as personal or professional relationships, affiliations, knowledge or beliefs) in the subject matter or materials discussed in this manuscript. 